\title{
The Use of Digital Applications and COVID-19
}

\author{
Arion R. Alexopoulos $^{1}\left[\right.$ [ Jake G. Hudson ${ }^{1} \cdot$ Oluwatomisin Otenigbagbe $^{1}$
}

Received: 22 June 2020 / Accepted: 18 July 2020 / Published online: 30 July 2020

(c) Springer Science+Business Media, LLC, part of Springer Nature 2020

\begin{abstract}
Mobile health apps are becoming increasingly popular amongst users who are turning to digital platforms to aid their mental wellbeing. As a result of the current COVID-19 pandemic, healthcare staff as well as recovering patients may suffer from PTSD. We have therefore suggested to Reyes et al. ("Promoting Resilience Among College Student Veterans Through an Acceptance-and-Commitment-Therapy App: An Intervention Refinement Study", 2020) the importance of repurposing their app to help these users to improve their emotional resilience and subsequently their ability to cope with the trauma of their experience. We have also discussed the most pertinent barriers to mobile health app uptake including data privacy concerns and the role of stigma.
\end{abstract}

Keywords Mental wellbeing applications $\cdot$ PTSD $\cdot$ COVID-19

We read with great interest "Promoting Resilience Among College Student Veterans Through an Acceptance-and-Commitment-Therapy App: An Intervention Refinement Study", Reyes et al. (2020). Our research group has investigated the barriers to user uptake of Mental Wellbeing Apps. Our study included conducting a survey of over 400 people encompassing a wide range of ages, ethnicities and backgrounds, (Alexopoulos et al. unpublished data). The investigation carried out led us to several interesting findings that we think are applicable to future research by Reyes et al. looking into PTSD.

Mobile health apps have become increasingly popular as society's focus shifts to the rapidly digitising era (Mikulic 2019) and within this there has been a rise in interest towards Mental Wellbeing apps (Bakker et al. 2016). Consequently, creating apps which are best suited to improving user experience while promoting mental wellbeing will become increasingly important.

Furthermore, the rapid changes that have followed the Coronavirus Pandemic have meant that provision of healthcare and mental wellbeing services is limited and may well continue to be difficult to access for a sustained period of

Arion R. Alexopoulos

ara215@ic.ac.uk

1 Imperial College London, Exhibition Rd, London SW7 2AZ, England, UK time as health services try to deal with the backlog that has arisen.

Importantly, amidst the chaos of this pandemic, the mental wellbeing of the frontline healthcare staff will be tested in these exceptional circumstances. Following the SARS-CoV epidemic in 2002-2004 "most" of the medical staff in A\&E departments in Taiwan were reported to have developed PTSD as a result of working on the front line in the fight against the virus (Torales et al. 2020). To compound this affect, there were already concerns with burnout and stress in healthcare staff pre-COVID-19 pandemic so this experience may well have an even greater impact on the mental wellbeing of front line staff (Yates 2020).

Moreover, research showed that patients who had recovered from MERS or SARS-CoV were themselves at an increased risk of developing longer term mental health conditions including PTSD. This has led to the conclusion that some patients are likely to go onto having PTSD secondary to their experience with COVID-19, a topic which has also garnered media attention in the recent weeks (Rodgers et al. 2020).

As a result, mental wellbeing apps-in particular those dealing with PTSD - may play a crucial role in helping healthcare workers to combat the adverse effects of working in such high pressure situations for a prolonged time period as well as improving outcomes of recovered COVID19 patients. We think that a study done to gain insight into where an app could aid healthcare workers or patients 
would be an excellent next area of research for Reyes et al. There could potentially be a significantly larger user group who would benefit from an interventional mobile application approach. Indeed, there has already been a successful implementation of an online intervention in Wuhan, where a group of scientists and clinicians have made a platform to provide support to healthcare staff working in affected hospitals (Cheng et al. 2020).

With regards to emotional resilience within healthcare staff, literature indicates that resilience plays an important role in improving mental wellbeing as well as reducing cognitive mistakes in the workplace (Murden et al. 2018). Research also suggests that PTSD sufferers can benefit from mobile application interventions and despite there being a lack of widespread clinical trials the initial results are promising, (Wickersham et al. 2019). Therefore, an app which promotes resilience in PTSD sufferers may be a crucial method of helping a large category of people who have been deeply affected by the current COVID-19 crisis.

Ensuring that the barriers to uptake for such an app have been addressed is of paramount importance in enabling users to engage with the app. Our research showed that stigma, concerns over data privacy and third party access to user data were key factors in preventing people from sharing data on mental wellbeing app platforms, (Alexopoulos et al. unpublished data). An area that would need to be further addressed is the impact of these factors specifically with veterans, healthcare workers and potentially recovered patients suffering from PTSD and using Reyes' et al. recommended app to ensure maximal user uptake and engagement.

Additionally, concerning the original cohort in Reyes et al. study, it would be valuable to investigate whether military veterans suffering from PTSD are more vulnerable at this time, with support and family and friends networks perhaps being more difficult to reach. This would be a novel area that could be researched to understand the true effects of COVID-19 on this patient group.

As discussed, there are several potential avenues that Reyes and his team could progress on with work related to their PTSD app in this unprecedented time. Perhaps the greatest impact of any future research could arise from investigating the uptake and effectiveness of this already developed app on healthcare workers and patients who have been directly or indirectly affected by the COVID-19 pandemic. This is certainly an area of interest for our research group in future work.

\section{Compliance with Ethical Standards}

Conflict of interest The authors declare that they have no conflict of interest.

Ethical Approval The opinions in this article are of the authors. No ethical approval was needed.

\section{References}

Bakker, D., Kazantzis, N., Rickwood, D., \& Rickard, N. (2016). Mental health smartphone apps: Review and evidence-based recommendations for future developments. JMIR Mental Health, 3, e7.

Cheng, P., Xia, G., Pang, P., Wu, B., Jiang, W., Li, Y., et al. (2020). COVID-19 epidemic peer support and crisis intervention via social media. Community Mental Health Journal, 56, 786-792.

Mikulic, M. (2019). Number of mHealth app downloads worldwide from 2013 to 2017. Retrieved May 12, 2020, from https://www. statista.com/statistics/625034/mobile-health-app-downloads/.

Murden, F., Bailey, D., Mackenzie, F., Oeppen, R. S., \& Brennan, P. A. (2018). The impact and effect of emotional resilience on performance: An overview for surgeons and other healthcare professionals. British Journal of Oral and Maxillofacial Surgery, 56(9), 786-790.

Reyes, A. T., Muthukumar, V., Bhatta, T. R., Bombard, J. N., \& Gangozo, W. J. (2020). Promoting resilience among college student veterans through an acceptance-and-commitment-therapy app: An intervention refinement study. Community Mental Health Journal. https://doi.org/10.1007/s10597-020-00617-4.

Rogers, J. P., Chesney, E., Oliver, D., Pollak, T. A., McGuire, P., FusarPoli, P., et al. (2020). Psychiatric and neuropsychiatric presentations associated with severe coronavirus infections: A systematic review and meta-analysis with comparison to the COVID-19 pandemic. The Lancet Psychiatry, 7, 611-627.

Torales, J., Higgins, M., Castaldelli-Maia, J. M., \& Ventriglio, A. (2020). The outbreak of COVID-19 coronavirus and its impact on global mental health. International Journal of Social Psychiatry, 4, 0020764020915212.

Wickersham, A., Petrides, P. M., Williamson, V., \& Leightley, D. (2019). Efficacy of mobile application interventions for the treatment of post-traumatic stress disorder: A systematic review. Digital Health, 5, 2055207619842986.

Yates, S. W. (2020). Physician stress and burnout. The American Journal of Medicine, 133(2), 160-164.

Publisher's Note Springer Nature remains neutral with regard to jurisdictional claims in published maps and institutional affiliations. 